\title{
March 2016 Pulmonary Case of the Month
}

\author{
Ramachandra R. Sista, MD \\ Maxwell L. Smith, MD \\ Lewis J. Wesselius, MD
}

Departments of Pulmonary Medicine and Pathology

Mayo Clinic Arizona

Scottsdale, AZ

\section{History of Present IIIness}

A 74-year-old man was referred for a recently identified right pleural effusion and dyspnea on exertion.

\section{Past Medical History, Family History and Social History}

He has a history of anemia, hypertension, and prostate cancer with a prostatectomy in 2015. He is a life-long nonsmoker and has no occupational exposures. Family history is noncontributory.

\section{Physical Examination}

He had diminished breath sounds at the right lung base and a palpable spleen.

Otherwise the physical examination was unremarkable.

\section{Laboratory}

CBC: hemoglobin $8.5 \mathrm{~g} / \mathrm{dL}$, white blood count $7.7 \times 109$ cells/L, platelets $357 \times 109$ cells/L.

\section{Radiography}

A chest X-ray showed a right pleural effusion. Representative images from the CT scan are shown in Figure 1.
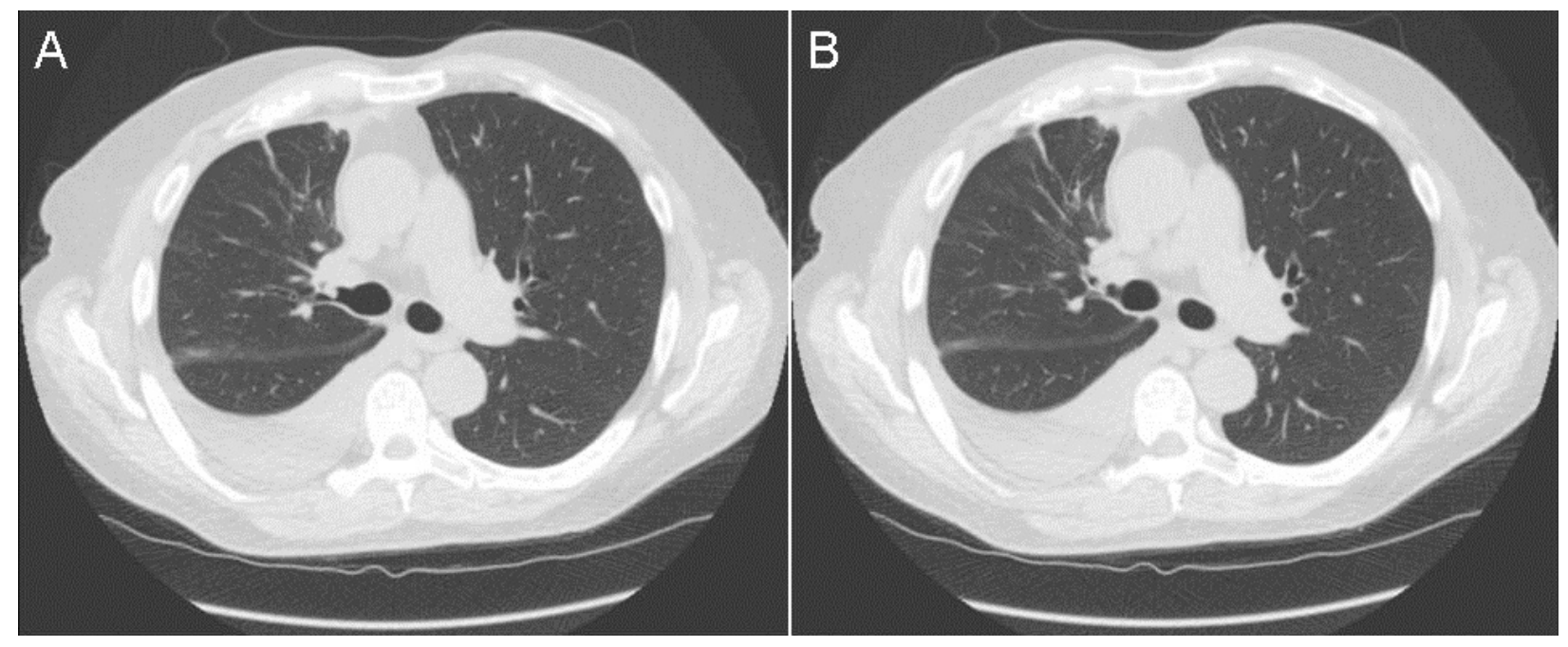

Figure 1. Representative images from the CT scan. 
Which of the following is the most likely diagnosis?
1. Empyema
2. Lung cancer
3. Tuberculosis
4. Usual interstitial pneumonia
5. Valley fever (coccidioidomycosis) 


\section{Correct! \\ 2. Lung cancer}

The CT scan of the chest shows an endobronchial lesion with the right pleural effusion (Figure 2).

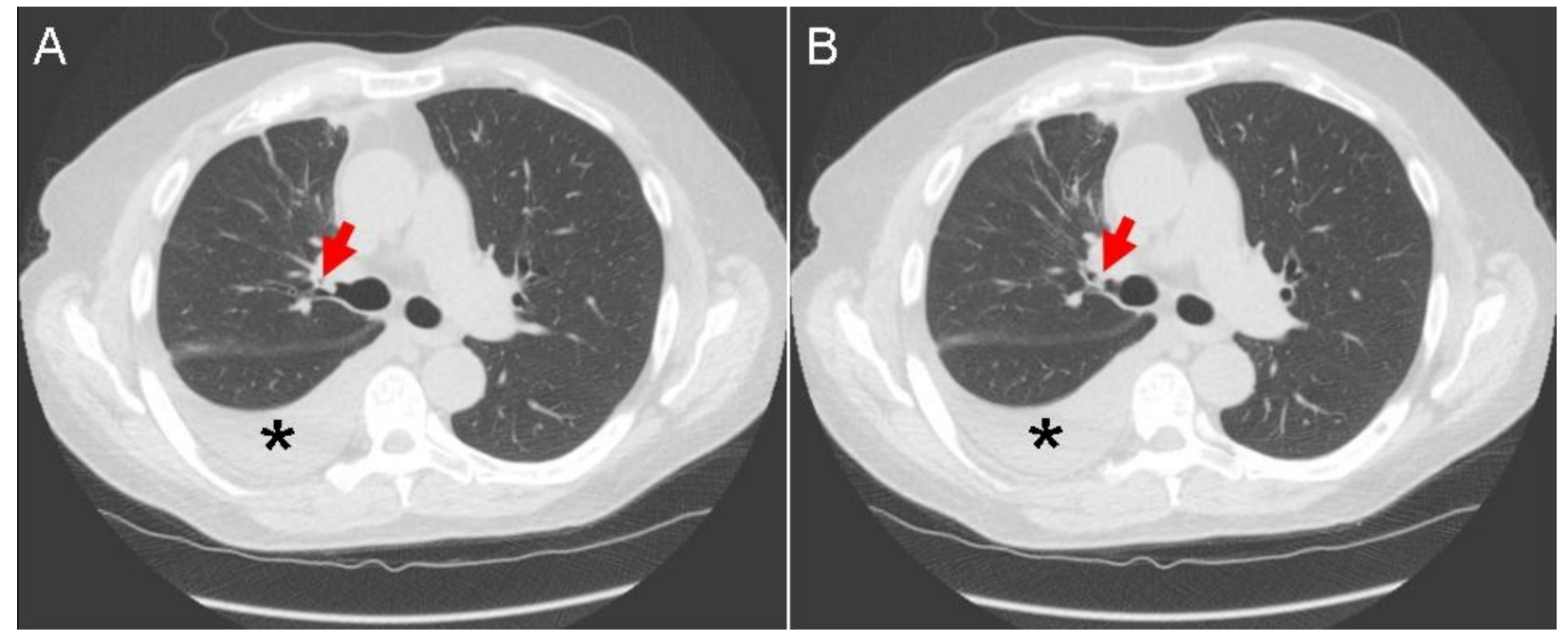

Figure 2. Representative images from the CT scan showing the right pleural effusion $\left(^{*}\right)$ and an endobronchial lesion (red arrows).

Which of the following is the next step in the patient's evaluation?
1. Bronchoscopy
2. Needle biopsy of the mass
3. Thoracentesis
4. 1 or 3
5. All of the above 


\section{Correct!}

\section{1 or 3}

Generally, we prefer to do a thoracentesis first because it can give a diagnosis of lung cancer metastatic to the pleura if malignancy is suspected. However, it would not be wrong to perform a bronchoscopy. The lesion is quite centrally located and bronchoscopy would be a much better choice for a biopsy.

A thoracentesis was performed. It revealed an exudative effusion with a protein 4.8 $\mathrm{g} / \mathrm{dL}, \mathrm{LDH} 363 \mathrm{U} / \mathrm{L}$, and a differential showing 93\% lymphocytes.

Although a pleural fluid cell differential is usually mostly macrophages and lymphocytes, a pleural fluid differential of $>80 \%$ lymphocytes is compatible which of the following diagnosis?

1. Lymphoma

2. Tuberculous effusion

3. Yellow nail syndrome

4. 1 and 3

5. All of the above 


\section{Correct! \\ 5. All of the above}

Although a lymphocyte predominant pleural effusion often suggests tuberculosis or malignancy, the differential diagnosis is quite large (Table 1) (1).

Table 1. Differential diagnosis of lymphocyte predominant pleural effusions.

- Acute lung rejection

- Chronic rheumatoid pleurisy

- Chylothorax

- Lymphoma

- Post-coronary artery bypass graft surgery (2-12 months)

- Sarcoidosis

- Tuberculous effusion

- Uremic pleural effusion

- Yellow nail syndrome

In this patient, the pleural fluid cytology was negative for malignancy and the cause of the pleural effusion was unclear. A bronchoscopy was performed, and an endobronchial lesion was encountered (Figure 3).

Figure 3. Right upper lobe endobronchial mass seen on bronchoscopy. 
Biopsies were performed of the lesion (Figure 4).

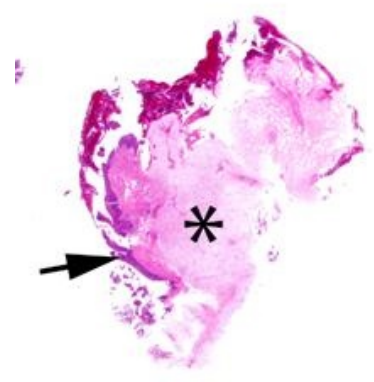

A
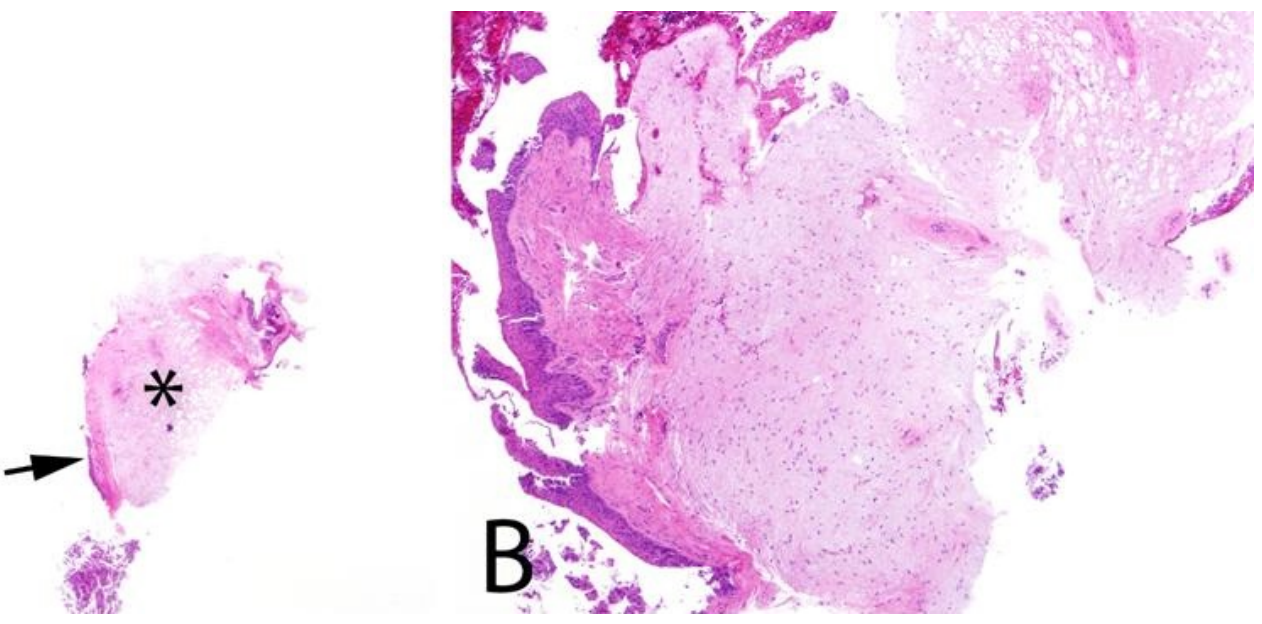

Figure 4. Panel A: Generous endobronchial biopsy fragments of tissue showing the surface epithelium (arrows) and a submucosal neoplastic proliferation (asterisks). H\&E, 20X. Panel B: Neoplasm in the submucosal space with mucopolysaccharide rich matrix typical of cartilage. H\&E, 100X.

Are $\underline{\text { metastases }}$ from the endobronchial lesion the cause of the pleural effusion?

1. No

2. Yes 


\section{Correct!}

\section{No}

The pathology diagnosis was endobronchial cartilaginous hamartoma, negative for malignancy. Therefore, the endobronchial lesion represent an incidental finding (2). Pulmonary hamartomas are the most common benign lung tumor, but only $1.4 \%$ are endobronchial, although some studies suggest higher incidence of $10-20 \%$. The tumors may be lipomatous or cartilaginous.

A bone marrow demonstrated large granular lymphocytic leukemia and the pleural effusion was attributed to diagnosis of lymphocytic leukemia with pleural involvement. The patient is currently being evaluated by hematology for possible chemotherapy.

\section{References}

1. Sahn SA. Getting the most from pleural fluid analysis. Respirology. 2012;17(2):2707. [CrossRef] [PubMed]

2. El-Kersh K, Perez RL, Gauhar U. A 63-year-old man with a chronic cough and an endobronchial lesion. Chest. 2014;145(4):919-22. [CrossRef] [PubMed] 